\title{
4. DER CVP 2945 (= HOLUBAŘGLOSSAR B)
}

Der originale Buchblock dieser Papierhandschrift enthält nach einem Vorsatzblatt mit noch erkennbaren, aber teils beschnittenen Schreibübungen - je zweimal angelus Engl, primus sexternus - weitere 83 Folien. Blatt 1 bis $47^{r}$, - mit alter Zählung - ist eine Kopie des ersten Teils von Holubařs Glossar, also das Abecedarium von Angelus bis Uxor, vgl. die Abb. 36 und 38. Die Schrift ist eine gute Minuskel des 15. Jahrhunderts mit roten Zierstrichen, auch der Beginn jeder Zeile ist rubriziert, am Beginn jeder Buchstabengruppe steht eine zweizeilige rote Initiale. Der beschriebene Teil der Handschrift trägt einen Raster zu je zwanzig Zeilen und drei Kolumnen, der leere Rest bis Blatt 83 eine blinde Kolumneneinteilung. Das Buch wurde leider nachträglich auf $205 \times 135 \mathrm{~mm}$ verkleinert und hat einen Pappeinband mit Vorsatz aus dem neunzehnten Jahrhundert ${ }^{130}$. Das Wasserzeichen, ein Anker mit Waage, ist nicht einzuordnen.

Es ist eine forschungsgeschichtliche Ironie, daß Zahradnik die Abschrift (B) nicht kannte, während umgekehrt Rudolf Wolkan, der zehn Jahre davor über den CVP 2945 geschrieben hatte, das Holubar̆glossar A nicht geläufig war ${ }^{131}$. Václav Flajšhans formulierte dann als erster den kopialen Zusammenhang beider Quellen ${ }^{132}$. Wie eng dieser aber wirklich ist, kann im Folgenden gezeigt werden.

Vergegenwärtigen wir uns zunächst die Texteinteilung: Wie im Holubařglossar A sollte auch im CVP 2945 das Abecedarium auf 48 Folien, dreispaltig zu je 20 Zeilen stehen ${ }^{133}$. Daß es ein Blatt weniger ist geht auf die Kappe des Kopisten: Nach $8^{r}$ übersah er zwei Seiten des Textes $\left(11^{\mathrm{v}}-12^{\mathrm{r}}\right)$, was nicht weiter auffiel, da mit $8^{\mathrm{v}}\left(=12^{\mathrm{v}}\right)$ der Abschnitt mit dem Buchstaben D beginnt. Häufig vergaß der Schreiber von B auch auf Einzelzeilen der Vorlage und suchte diese nach Möglichkeit später auf gleicher Seite einzufügen, vgl. $4^{\mathrm{r}}\left(=7^{\mathrm{r}}, \mathrm{Z} .9\right.$, wobei er in die Zeile davor die tschechische Entsprechung von $7^{\mathrm{r}}$, Z. 9 einfügte), vgl. auch $\boldsymbol{\gamma}^{\mathrm{v}}\left(=10^{\mathrm{v}}\right.$, Z. 5/6) mit zweimaligem promieniti. Auf $8^{\mathrm{v}},\left(=12^{\mathrm{v}}\right.$, Z. 9) wiederholt A irrtümlich Decima, was dem Kopisten vorerst nicht auffiel, sodaß er ab $9^{\mathrm{r}}\left(=13^{\mathrm{r}}\right)$ bis $12^{\mathrm{v}}\left(=17^{\mathrm{v}}\right)$ A um eine Zeile nachläuft. Dieses Mißverständnis wiederholt sich, wie noch gezeigt werden wird, auch bei allen anderen Zeilendopplungen lateinischer Leitwörter in A $\left(17^{\mathrm{v}}, \mathrm{Z} .4,42^{\mathrm{r}}, \mathrm{Z} .14,47^{\mathrm{r}}, \mathrm{Z}\right.$. 12). Auf $12^{\mathrm{v}}$ ( $=17^{\mathrm{v}}$, achtzehnzeilig) ergibt sich dann eine gröbere Störung, weil A Exacerbare der Zeile 4 im nächsten System wiederholt, B auf die Wortgleichung Excutere/ Auzslan/ witizisti vergißt und diese auf der nächsten Seite nachholt. Dadurch ergibt sich im CVP 2945 Platz für drei Zeilen auf zwanzig, vgl. 18 ${ }^{\mathrm{r}}$ Z. 1-3. Auf $1^{\gamma^{\mathrm{v}}}\left(=21^{\mathrm{v}}\right)$ wird Generare - man

130 Beschreibung in K. Schwarzenberg, Katalog der kroatischen, polnischen und tschechischen Handschriften der Österreichischen Nationalbibliothek (Museion N.F. 4/4). Wien 1972, 16.

131 Wolkan, Geschichte der deutschen Literatur (zit. A. 91) 65.

132 FlajšHans, Klaret (zit. A. 75) XXX.

133 In allen weiteren Erörterungen - also etwa auch im Kapitel 9 - steht A für das Glossar Holubařs, B für den CVP 2945. Nur in der folgenden Zusammenstellung dieses Kapitels ist, soweit es um die Erörterung formaler Diskrepanzen geht, auch die Blattzählung getrennt angegeben. Kursive Folienangaben beziehen sich auf B, gerade, (die für unsere Edition maßgebend sind), auf A. 
möchte sagen routinemäßig - am Schluß der Seite nachgeholt. Danach ist die Kongruenz wiederhergestellt. Auf $20^{\mathrm{v}}\left(=24^{\mathrm{v}}\right)$ kommt es zu zwei Zeilenvertauschungen, die Störung könnte durch die graphische Ähnlichkeit von Imperator/Imperare und Impetrare/Imperitus ausgelöst sein. Die Einträge $22^{\mathrm{v}}\left(=26^{\mathrm{v}}, \mathrm{Z} .12\right)$ und $23^{\mathrm{v}}\left(=27^{\mathrm{v}}, \mathrm{Z} .17\right)$ wurden glattweg vergessen, wodurch sich auf $24^{\mathrm{r}}\left(=28^{\mathrm{r}}\right)$ ein Vorlauf von einer Zeile ergibt. Das Gleichgewicht wird wieder hergestellt, indem B einfach den letzten Eintrag dieser Seite ausläßt. Auf $38^{\mathrm{r}}$ $\left(=42^{\mathrm{r}}, \mathrm{Z} .14\right)$ wiederholt A Sepelire als neue Zeile, worauf B prompt hereinfällt und die erste Zeile von $42^{\mathrm{v}}$ noch auf $38^{\mathrm{r}}$ schreibt. Fortan ergibt sich bis $42^{\mathrm{r}}\left(=46^{\mathrm{r}}\right)$ wieder ein Vorlauf von einer Zeile und eine kleinere Störung auf $39^{\mathrm{v}}\left(=43^{\mathrm{v}}, \mathrm{Z}\right.$. 11). Die Verschiebung verleitet den Schreiber übrigens zur Dopplung Rzecz am Beginn von $43^{\mathrm{r}}$ bzw. 39r . Das Spiel, daß den Kopisten von B einem Fehler in A aufsitzen ließ, wiederholt sich auf $43^{\mathrm{r}}\left(=47^{\mathrm{r}}, \mathrm{Z} .12\right)$, wo A das lat. Lemma Tinea in der nächsten Zeile repetiert. Wieder läuft B - mit kleiner Zeilenstörung auf $44^{\mathrm{r}}\left(=48^{\mathrm{r}}\right)$ - bis $45^{\mathrm{v}}\left(=49^{\mathrm{v}}\right)$ voraus, vergißt dort auf Zeile 2 , holt aber den ersten Eintrag von $50^{\mathrm{r}}$ noch auf das alte Blatt. Somit eilt erstmals A von $46^{\mathrm{r}}\left(=50^{\mathrm{r}}\right)$ bis $46^{\mathrm{v}}\left(=50^{\mathrm{v}}\right)$ voraus. Der Schluß $\left(4 \%^{\mathrm{r}} .=51^{\mathrm{r}}\right)$ ist wieder kongruent.

In einigen Fällen irrtümlicher Kolumnen- oder Zeilenvertauschung korrigierte der Schreiber seinen Irrtum selbst durch Merkzeichen, vgl. $13^{\mathrm{v}}\left(=17^{\mathrm{v}}, \mathrm{Z} .14\right), 1^{\mathrm{F}^{\mathrm{r}}}\left(=21^{\mathrm{r}}, \mathrm{Z} .6\right)$ und $28^{\mathrm{v}}$. $\left(=32^{\mathrm{v}}\right.$, Z. 5 bzw. 8), 33 $\left(=37^{\mathrm{r}}\right.$, Z. 8/9). Auch Lemmatavertauschung zwischen deutscher und tschechischer Kolumne hat er von der Vorlage übernommen, vgl. $18^{\mathrm{r}}\left(=22^{\mathrm{r}}, \mathrm{Z} .1\right)$ bzw. $22^{\mathrm{r}}\left(=26^{\mathrm{r}}, \mathrm{Z} .7\right.$ ), wobei das zweite Beispiel zeigt, wie sklavisch er der Vorlage zu folgen suchte: Statt Alzo/Tak steht Tak/Alzo, er hatte allerdings richtig begonnen, wie ein getilgtes $a l$ vor Tak belegt. Auf 50 , Z. $9\left(=46^{\mathrm{r}}\right)$ fehlt in A die deutsche Übersetzung, das tschechische Nawssczewiti für Uisitare ist daher in die zweite Kolumne vorgerückt. Genauso schreibt der Kopist, ein Korrektor, von dem gleich zu hören sein wird, hat dann kursiv haymsuchen ergänzt. Auf $7^{\mathrm{v}}$, Z. 3 fehlen A und B die vulgärsprachlichen Entsprechungen zu lateinischem Avriga, auf 34r Z. 13 Palma/Tener haben beide kein tschechisches Gegenstück. Auf 39r

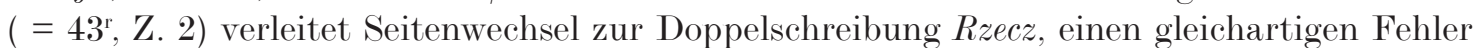
Blazen hat der Skribent auf $41^{\mathrm{r}}\left(=45^{\mathrm{r}}, \mathrm{Z}\right.$. 4) wieder getilgt, vgl. auch doppeltes auffganck $\left(33^{\mathrm{v}}\right.$, Z. 11/12) bzw. Cztyri $\left(38^{v}\right.$, Z. 3/4) oder die Vertauschungen der deutschen Kolumne auf $43^{\text {r, }}$ Z. 16/17. Ein Mißverständnis durch die Vorlage führte auch zur Zeilenkorrektur auf $40^{\mathrm{v}}\left(=44^{\mathrm{v}}\right.$, Z. 16/17). In $26^{\text {v }}$, Z. 11 steht in der tschechischen Kolumne fälschlich layk, das er von der unterschlagenen Folgezeile Laycus/leige/layk irrtümlich übernommen hat. Nach alldem ist nicht zu verwundern, daß auch die falsche deutsche Umsetzung Egil (17 $7^{\mathrm{r}}$ Z. 3, vgl. S. 31) in B wieder auftaucht.

Diese Beispiele formaler Art belegen eine unmittelbare Abhängigkeit des CVP 2945 von A, von dem auch sämtliche auf S. 31 f. erläuterten Störungen der alphabetischen Anordnung weitergegeben wurden. Der Kopist hat sich - schon aufgrund des vorgegebenen zwanzigzeiligen Rasters - bemüht, formal deckungsgleich mit der Vorlage zu arbeiten und übernahm nebenbei auch sämtliche im Kapitel über die Handschrift A beschriebenen Nachträge des vergleichbaren Vorlagetextes.

Ein Blick auf unsere synoptische Edition genügt, um die Differenzen von B zu A in den einzelnen Sprachkolumnen zu erkennen. Der lateinische Teil ist - wie zu erwarten - praktisch gleichgeblieben, und den engen Anschluß an A belegen gleichartige Fehler: Aderere (6r, Z. 6) Expungnare (18 ${ }^{\mathrm{r}}$, Z. 8), Fasilis (18v, Z. 5), Diuininitas $\left(14^{\mathrm{v}}, \mathrm{Z} .17\right)$, Hibaris $\left(22^{\mathrm{v}}\right.$, Z. 19), Ligua bzw. Ligwa (27v', Z. 15), he (29r, Z. 7), Nummulary statt Nummularius (32', Z. 9), Posidere (37",

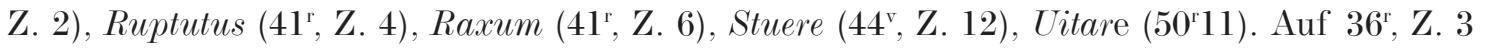
kann B mit Peruertit(us) ein Peruertir von A nicht richtigstellen, Auf $36^{r}, \mathrm{Z} .3$ kann B mit 
Pervertit(us) ein Peruertir der Vorlage nicht richtigstellen. Dazu kommen eigenständiger Unachtsamkeiten beim Kopieren, z. B. Angnus (4r, Z. 18), Anwilla (4v, Z. 17), Barbar statt Barba (7v, Z. 15), Delinquere statt Derelinquere (13 $\left.{ }^{\mathrm{r}}, \mathrm{Z} .18\right)$, Eicire statt Eicere (16 $\left.{ }^{\mathrm{r}}, \mathrm{Z} .8\right)$, Exutacio statt Excitatio (18 , Z. 18), Laticare statt Latigare (27 ${ }^{\mathrm{r}}$, Z. 9), Metidacio (29 ${ }^{\mathrm{r}}$, Z. 15), Nominati (us) (31 $\left.{ }^{\mathrm{v}}, \mathrm{Z} .17\right)$, Pedere statt Perdere $\left(35^{\mathrm{v}}, \mathrm{Z} .3\right)$, Repellere statt Replenere (39', Z. 9), Susurrare statt Suspirare in A (45, Z. 20), Ppranum statt Pravum $\left(37^{\mathrm{v}}, \mathrm{Z} .11\right)$, Redartare $\left(39^{\mathrm{v}}\right.$, Z. 5), Sesdere statt Sedere (41v, Z. 16) usw. In einigen Fällen hat B aber auch gebessert, vgl. Concupisciencia $\left(10^{\mathrm{v}}, \mathrm{Z} .13\right)$, expellere $\left(18^{\mathrm{r}}, \mathrm{Z} .3\right)$ usw. Auf 18 $8^{\mathrm{r}}, \mathrm{Z} .7$ schrieb der Kopist zunächst richtig Exprobare, übernahm aber dann durch $r$-Überschreibung zu Exprobrare den Irrtum der Vorlage. Auf 22r, Z. 1 wollte er Globus durch die Nebenform Glomis ersetzen, dabei kam - als typischer Kopierfehler - Glouuis heraus, vielleicht ein Hinweis, daß neben der Vorlage noch andere Hilfsmittel zur Verfügung standen.

Auch die tschechische Kolumne bleibt im Vergleich zu A relativ konstant, was wohl damit zu tun hat, daß das Tschechische, als erste stabilisierte slawische Schriftsprache, im 15. Jahrhundert graphematisch fester war als das Deutsche ${ }^{134}$. Wieder werden etliche Fehler von A übernommen, vgl. Ager/Bole $\left(6^{\mathrm{r}}, \mathrm{Z} .18\right)$, ztraczny, das sogar zu ztraczwy wird $\left(12^{\mathrm{v}}, \mathrm{Z} .3\right.$, tschechisch ztracený), Studicze(n) (20', Z. 12), Boboyowati (24, Z. 16), Odolati (40v , Z. 18), Ostastati (40 ${ }^{\mathrm{r}}$ Z. 10), Cziti $\left(42^{\mathrm{r}}, \mathrm{Z} .7\right)$, Strzi $\left(43^{\mathrm{v}}, \mathrm{Z} .10\right)$. Allerdings wird Koh zu Roh korrigiert $\left(38^{v}, Z\right.$ Z. 2). Weitere Fehler ergaben sich weil B interlineare Korrekturen von A übersehen hat, vgl. Ssptati statt Sseptati(45v Z. 20). Auch die übrigen Varianten sind zumeist Abschreibfehler, vgl. krathy ( $\left.8^{\mathrm{r}}, \mathrm{Z} .10\right)$, neyaswy statt neyasny (12 ${ }^{\mathrm{v}}$, Z.17), zahbatiti statt Zahladiti (13 ${ }^{\mathrm{r}}, \mathrm{Z}$. 3), Rzieklsein statt Rziekl sem (14r, Z. 2), Sstzatny statt Sstzastny (20v, Z. 7), Reprzitel statt Neprzitel (24 ${ }^{\mathrm{v}}$, Z. 7), Dluhowczny statt Dluhoweczny (28, Z. 8), placet statt placek (28 ${ }^{\mathrm{r}}$, Z. 15), przwozeni statt Przirozeni $\left(31^{\mathrm{r}}, \mathrm{Z} .13\right)$, modlitha $\left(33^{\mathrm{v}}, \mathrm{Z} .5\right)$, wohl auch hrzifrak statt Hrzissnik (35, Z. 6), putrack statt Putnik (35', Z. 5), nasledowruck statt Nasledownik (35', Z. 17), ferner Noby statt Nohy (35, Z. 9), Rowe statt Rowne $\left(36^{\mathrm{v}}\right.$, Z. 5), Tiknuti statt Tisknuti $\left(38^{\mathrm{r}}\right.$, Z. 3), podke statt Podle (41 v', Z. 9), Sednu statt Gednu (41'v, Z. 20), pekho statt Peklo (46', Z. 13), Zad statt $\mathrm{Had}\left(50^{\mathrm{r}}, \mathrm{Z} .2\right)$, v.a. aber die $r$ - $i$-Verlesungen, weil der Kopist die $r z$-Ligatur seiner Vor-

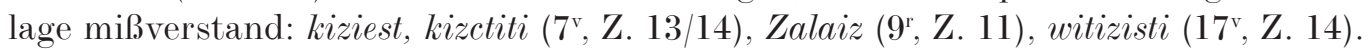

Trotz solcher Schnitzer dürfte der Kopist bzw. Bearbeiter doch irgendeinen slawischen Hintergrund gehabt haben. Dafür sprechen zunächst ein li dam statt Dabo/Dam (12v , Z. 1), die digraphischen Schreibungen Zagikaway statt Zagikawy (7v, Z. 11), Ssedwoy statt Ssediwy $\left(9^{r}\right.$, Z. 6), zadostiwoy statt Zadostiwy $\left(13^{v}\right.$, Z. 4) oder die Verkleinerungsform von Pytel zu pytek (42 $2^{\text {r }, ~ Z . ~ 19) . ~ A u f ~ 39 r, ~ Z . ~} 12$ ist winiti für Reprehendere zu wirati variiert. Das Nomen für Macula/Poskwrna wird zu Poskwati $\left(28^{v}\right.$, Z. 7), Regina/Kralowna ebenfalls fälschlich zum Verb kralowati $\left(40^{\text {r }, ~ Z . ~ 6) . ~}\right.$

Die Änderungen im deutschen Text von B sind so vielfältig, daß sie hier nur zusammenfassend als Bavarisierung einer mitteldeutschen Vorlage charakterisiert werden können. Allerdings hat B dabei nicht konsequent durchgegriffen, so zwar, daß sogar von den im Zusammenhang mit dem Ladislauskodex genannten Beispielen mhd. Langvokale noch immer

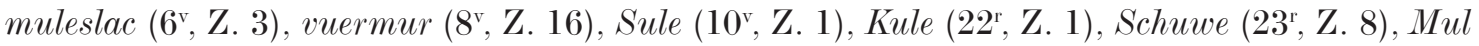

134 S. E. Skála, Deutsch und Tschechisch im mitteleuropäischen Sprachbund, in: M. Berger - K. Krolop, Brücken (= Germanistisches Jahrbuch 1991/92). Berlin-Prag-Prerau 1992, 173-179 bzw. Vintr, Das Tschechische (zit. A. 13) 153. Vgl. dazu wohl auch V. BoK, Slovník středověké něměiny pro historiky. Budweis 1995, S.19. 


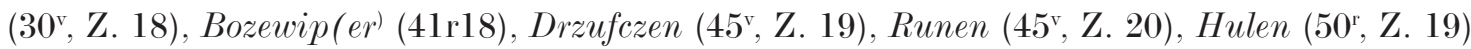
überlebten und glichin erst nachträglich zu gleichen korrigiert wurde $\left(10^{\mathrm{r}}, \mathrm{Z}\right.$. 10). Umgekehrt überrascht nicht, daß B die mitteldeutsche Monophthongierung meist digraphisch auflöst, vgl. dipp zu dieb $\left(21^{\mathrm{r}}, \mathrm{Z} .16\right)$, Gisen zu Giessen $\left(21^{\mathrm{r}}, \mathrm{Z} .13\right)$, Cruk zu krueg $\left(7^{\mathrm{r}}\right.$, Z. 7), Hute zu Hüett $(9$ v Z. 1) usw. Entsprechend geändert werden auch die mitteldeutschen Umlautungen von mhd. ou, vgl. kauffen gegen keufen $\left(16^{\mathrm{r}}, \mathrm{Z} .19\right)$, enhauppen gegen enteupten (!) (12v, $\left.\mathrm{Z} .12\right)$, Versaum $(m)$ en gegen Virzeumen $\left(31^{v}, Z\right.$. 1) u.a. Auch herrscht eine Tendenz, mhd. ei als ay, ai zu realisieren, vgl. 15, Z. 2 B Zway A Czwey, 15 $5^{\mathrm{v}}$ Z. 9 B helffe(n)pain A Helfenbein, wobei auch mitteldeutsches e betroffen ist: $34^{\mathrm{v}}, \mathrm{Z} .7$ bzw. 37, Z. 18 B ayntayl A enteil usw., dazu hyperkritisches schaydtel aus Medula/schedel $\left(29^{r}, Z\right.$ Z. 16). Die augenfälligste Änderung betrifft aber mitteldeutsches i in Nebensilben, vgl. erlosen statt Erlosin ( $\left.5^{\mathrm{r}}, \mathrm{Z} .7\right)$, hocher statt hohir

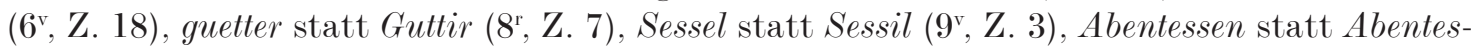
$\sin \left(9^{\mathrm{v}}, \mathrm{Z} .10\right)$, verspotten statt Virspattin $\left(13^{\mathrm{r}}, \mathrm{Z} .18\right)$ usw. Die mitteldeutschen cz für Affrikata werden vielfach vereinfacht, vgl.Zwpintten gegen czubinden $\left(6^{v}, Z\right.$. 14), zwir gegen Czwir $\left(8^{r}, Z\right.$. 6), wolgezogen ( $\left.n\right)$ gegen Wolgeczogen usw., die häufige mitteldeutsche z-Schreibung für s wird zurückgenommen, vgl. haws zu huz (15, Z. 10), Sey zu Zei $\left(17^{\mathrm{r}}, \mathrm{Z} .10\right)$, eyssen zu Eyzen

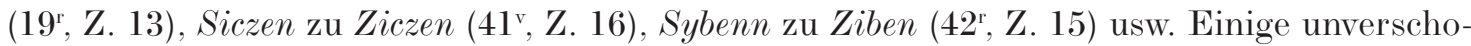
bene Formen werden immerhin übernommen, vgl. Creator/Schepper $\left(11^{\mathrm{v}}, \mathrm{Z} .13\right)$, Nepczen $15^{\mathrm{r}}$, Z. 14, zu mhd. nafzen), snarken (44', Z. 17), eventuell Strepitus/Getreplt (44 ${ }^{\mathrm{v}}, \mathrm{Z}$. 20). Auf $10^{\mathrm{r}}$, Z. 11 wurde richtiges Cophinus/korp falsch zu kropff, wobei der Schreiber vielleicht hyperkritisch dachte und seinem Hang zu methathetischen Schreibungen aufsa $\beta^{135}$.

Natürlich ist die Bavarisierung auch in der ,südlichen“ Wortwahl sichtbar, hier seien nur Zütutler statt Zublezer (6r, Z. 12), chirczen statt Luchte (8v , Z. 20), gayss statt Czige (9 ${ }^{\mathrm{r}}$, Z. 9), Keychen statt kerker (9', Z. 11), zand statt Czan (13', Z. 10), hiert statt Herte (15 $\left.{ }^{\mathrm{v}}, \mathrm{Z} .5\right)$, haffner statt Toppher (19v, Z. 8), Scheffman statt Schiffman (31' Z. 15), gegen statt kegen (32 $\left.{ }^{\mathrm{v}}, \mathrm{Z} .18\right)$, Bischolf statt Bischof bzw. Bischalf statt Bischaf $\left(16^{\mathrm{v}}\right.$, Z. 8, 37 $7^{\mathrm{r}}$, Z. 9), Hafftel statt Virspan (recte Virspang,30v, Z. 6), gegn(n)wurtig statt Kegenwertig $\left(38^{r}\right.$, Z. 5), Sambstag statt Zunnabent $\left(42^{\mathrm{r}}\right.$, Z. 16), Holler statt holunder $\left(42^{\mathrm{v}}\right.$, Z. 6), denckhant statt lenkhant $\left(43^{\mathrm{v}}, \mathrm{Z} .8\right)$ und zumindest sinngemäß auch taffern $(n)$ statt Kreczem $\left(46^{r}, Z\right.$ Z 1) angeführt. Darüberhinaus gibt es noch andere interessante Synonymwechsel und Varianten, etwa bestatten für Pfesten/ Confirmare (10v , Z. 16), treugnus für Decepcio/Trugeheit (12v, $\mathrm{Z} .10)$, erlosen für Delibe (r)are/ Betrachten (!) (13 $\left.{ }^{\mathrm{r}}, \mathrm{Z} .4\right)$, geschmeydikait statt Bescheid(en)heit $\left(14^{\mathrm{v}}, \mathrm{Z} .1\right)$, lob statt Gutnam (18v , Z. 12), Mayligen statt Vnfletiken (28v , Z. 8), Gedancknus statt Gedechtnus (29', Z. 19), Geyer statt weige (30, Z. 2), durftik statt arm (30 ${ }^{\mathrm{r}}$ Z. 13), Schafstal statt schofhuz $\left(34^{\mathrm{r}}, \mathrm{Z} .3\right)$, geuelt statt Beheit $\left(36^{r}\right.$, Z. 20), widerbrengen statt widergeben $\left(39^{r}, Z\right.$ Z. 11), astt statt Enczwike

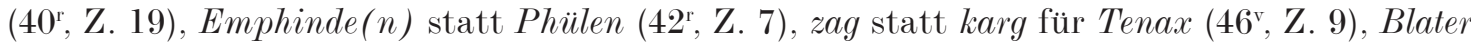
statt Blaze (49', Z. 19).

Einige bemerkenswerte semantische Änderungen in der deutschen Kolumne von B stammen von der Hand eines Korrektors, dessen kursive Ergänzungen und Besserungen, die sich allerdings nur auf einige Blätter beschränken, in der Edition besonders vermerkt sind (vgl. auch Abb. 37). Hier nur einige Beispiele: Arare/Eren (5v , Z. 4, so von A übernommen) wird zu Egen, auf 5 ${ }^{\text {v }}$ Z. 14 tilgt er Sprechen als falsche Umsetzung von Aspergere und schreibt - wie A - richtig Sprengen, ersetzt Reinen durch erleiwtern (n) $\left(16^{\text {v }}\right.$, Z. 3), Euacuare/Ausbeduten durch ausleiten(n) (16, Z. 4), Rechtikeit durch pillikait (16 $\left.{ }^{\mathrm{v}}, \mathrm{Z} .12\right)$, Erectus/Aufgericht durch

${ }^{135}$ Vgl. dazu S. 77. 
erhebt (16 $\left.6^{\mathrm{v}}, \mathrm{Z} .14\right)$, Ropczen durch aufruckn(n) (17 $\left.7^{\mathrm{r}}, \mathrm{Z} .1\right)$, ergänzt bei euadere/Abkummen fliehen $(n)\left(17^{\mathrm{r}}, \mathrm{Z} .15\right)$, ersetzt Topf durch heffn $(n)\left(33^{\mathrm{r}}, \mathrm{Z}\right.$. 13), Derleczt durch newist $\left(32^{\mathrm{r}}\right.$, Z. 1), Eczen durch neren (32r, Z. 17) usw. Vereinzelt kann man seine Wortsuche in mehreren Etappen nachvollziehen: 16 ${ }^{\mathrm{v}}$, Z. 4 schreibt A für Euacuare Auzbeduten, darüber ledigen. Der Korrektor in B hat Ausbetutten bzw. ledigen getilgt und schreibt darüber ausleiten $(n)$. Auf 35 ${ }^{v}$, Z. 17 Persecutor setzt er zu Volger betrieg(er) und v(er)ate(r), das er wieder streicht. Einen Fehler mit Vertauschung der deutschen Lemmata begeht er auf 17 $7^{\mathrm{r}}, \mathrm{Z} .17 / 18$.

Übrigens zeigen die vielen Fehlschreibungen des Kopisten von B, daß er eines sehr viel genaueren Korrektors bedurft hätte. Zunächst - und das belegt den unmittelbaren Abschriftcharakter nach A - übernimmt er viele Mißverständnisse der Vorlage, hier seien nochmals Balbus/Stamelder (7 , Z. 11), Delicatus/A eliczart man B elizzartman (13 $\left.{ }^{\mathrm{r}}, \mathrm{Z} .5\right)$, Eneus/AB Messigen (16 ${ }^{\mathrm{v}}$, Z. 6), Schuwe $\left(23^{\mathrm{r}}\right.$, Z. 8) und Quocumq(ue)/AB Wonorthin $\left(38^{\mathrm{v}}\right.$, Z. 20), Envude $\left(38^{\mathrm{r}}, \mathrm{Z}\right.$. 15), weiters Fletus/A weiunge B waiunge $\left(20^{\mathrm{r}}, \mathrm{Z} .6\right)$, bzw. - fehlgelesen - weiunge $\left(28^{\mathrm{r}}\right.$, Z. 15), Wolliclichen statt Wonniclichen (22v, Z. 5), slipset statt slipfet $\left(28^{\mathrm{r}}, \mathrm{Z} .11\right)$, enstillen $\left(30^{\mathrm{r}}\right.$, Z. 17), Envude (38', Z. 15), Reliquie/A heilittum B hellitum (40', Z. 9), Succidere/AB vnd(er)schniedn(n) (45 , Z. 14), Superius/Vben (45 $\left.{ }^{\mathrm{v}}, \mathrm{Z} .4\right)$, Taxare/Schacze (46 ${ }^{\mathrm{r}}, \mathrm{Z}$. 16), Tela/A Leiment B lennent $\left(46^{\mathrm{v}}, \mathrm{Z} .2\right)$ und Torrens/flis $\left(47^{\mathrm{v}}, \mathrm{Z} .2\right)$ genannt. 10 ${ }^{\mathrm{r}}, \mathrm{Z}$. 13 variiert er die Störung von A Cognatus/Nefte zu neste. Auf 25v, 2.2 übernimmt er von A zunächst falsches Indigencia/Durfur, das er nachträglich zu Durfen bessert. Aber auch richtige Eintragungen des Ladislauskodex versteht er nicht: So wird gleich das erste doppelstöckige w der Vorlage zum Problem, so daß er anvrawe mit u-Überschreibung des $w$ zu anvra $(u)$ de mit u-Häkchen (?) über dem $r$ verschlimmbessert $\left(4^{\mathrm{r}}, \mathrm{Z} .10\right)$, Virdrucken wird zu vnd(er) rucken (12v, Z. 13), Derslaen zu derschbergen (31 $\left.{ }^{\mathrm{r}}, \mathrm{Z} .17\right)$, Orphanus/Enweiz zu Erweiz (33 $\left.{ }^{\mathrm{v}}, \mathrm{Z} .14\right)$, Gevnrei $(n) t \mathrm{zu}$ gewren (37 ${ }^{\mathrm{r}}$, Z. 2), Rotfüchsichter zu Rotschuchtige(r) (41 ${ }^{\mathrm{r}}, \mathrm{Z}$. 2), Gemitus/Zufzung zu Zussrung $\left(21^{\mathrm{v}}, \mathrm{Z} .8\right)$, Monere/Monen $\mathrm{zu}$ wanen $\left(30^{\mathrm{v}}\right.$, Z. 14), Uescitur/Erschpeizet sich zu Erschperzetsich $\left(49^{r}\right.$, Z. 18) mit der bereits in tschechischen Beispielen gezeigten r-i-Verwechslung. Wie dort übersieht er auch Überschreibungen von A, vgl. fleich gegen fleisch (9r, Z. 14), vnschmachait gegen Virsmeheit $\left(11 \mathrm{r}^{1}, \mathrm{Z} .1\right)$.

Dazu kommt eine große Zahl von neuen Flüchtigkeitsfehlern: frewtdin statt frewntdin (4 $\left.4^{\mathrm{r}}, \mathrm{Z} .12\right)$, Acceptus/Chenem statt Genem (5 $\left.{ }^{\mathrm{r}}, \mathrm{Z} .10\right)$, endenckenn statt entecken (13 $\left.{ }^{\mathrm{v}}, \mathrm{Z} .11\right)$, zoglel statt zogel $\left(9^{\mathrm{r}}, \mathrm{Z} .19\right)$, vogehus statt vogelhus $\left(9^{\mathrm{r}}, \mathrm{Z} .20\right)$, abgesprochn(n) statt Hab gesprochn(n) (14 $\left.{ }^{\mathrm{r}}, \mathrm{Z} .2\right)$, dassebig $\left(15^{\mathrm{v}}, \mathrm{Z} .6\right)$, enphilen statt Effugere/Enphlien (16 $\left.{ }^{\mathrm{r}}, \mathrm{Z} .2\right)$, ausnemnien statt Auznemen 16 ${ }^{\mathrm{v}}$, Z. 18), geschickt statt Geschicht (17\% ${ }^{\mathrm{r}}$ Z. 18), Snidt statt Smidt $\left(18^{\mathrm{v}}\right.$, Z. 2), Gewrein statt Gev(n)reint $\left(37^{\mathrm{r}}, \mathrm{Z} .2\right)$ usw. Bisweilen bessert er aber auch, vgl. etwa B Ardet/prindt A Bruet (5 , Z. 7), B armbrust/ A arbrust (7, Z. 12), Cessa/ B lashin A Lasin

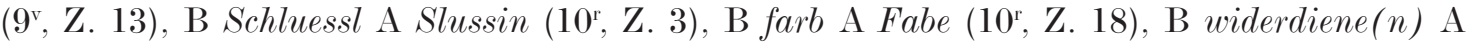
Virdien (13v, Z. 2), B ausziechen A Auzczhen (18 ${ }^{\mathrm{r}}$, Z. 19), Fossa/Eingraben gegen Eingrub $\left(20^{\mathrm{v}}\right.$, Z. 8), Fragmenta/Prossen gegen Bozem (20, Z. 12), Grex/hert gegen her (22r, Z. 15), Frashait statt Geiczickeit für lateinisch Gula (22r, Z. 20), B wang A wanle, B (29r, Z. 10), Der allergroste A Allergroser $\left(29^{\mathrm{r}}, \mathrm{Z} .10 / 11\right)$, B thuen A Tum (35', Z. 15), B Streitt A Sreit $\left(38^{\mathrm{r}}, \mathrm{Z} .2\right)$, B warvmb A warm (38', Z. 8), B anttwurt A Atwort (39ㄷ, Z. 2), B Temptare/v(er)Suechn(n) A Verzichen $\left(46^{\mathrm{v}}\right.$, Z. 7), B Schwindl A Schesind (49두 Z. 17) B wittib A witwa (49v Z. 11) u.a.

Zusammenfassend läßt sich zunächst feststellen, daß B - v. a. aufgrund formaler Abhängigkeiten und der vielfachen Übernahme von Fehlern in allen drei Sprachen - eine unmittelbare Kopie von A sein muß. Dafür sprechen die früher genannten formalen Gründe und rein optisch bedingte Kopierfehler. Signifikant ist ferner, daß - im Gegensatz zu anderen handschriftlichen Weitergaben von Glossartexten - B als jüngere Überlieferung keinerlei Zusätze, 
wohl aber durch ungenaue Abschrift bedingte Ausfälle gegenüber A aufweist, vgl. $11^{\mathrm{r}}$ bis $12^{\mathrm{v}}$ bzw. $26^{\text {v }}$, Z. 12 und $27^{\text {v }}$, Z. 17.

Der deutsche Text ist übrigens so wenig konsequent bavarisiert, daß Rudolf Wolkan wie bereits im vorvorigen Kapitel zitiert - den ostmitteldeutschen Hintergrund hervorheben konnte. Der Bearbeiter dürfte - neben seinen bairisch-österreichischen Deutschkenntnissen - zumindest einigermaßen auch Latein und Slawisch verstanden haben. Darüberhinaus fällt es schwer, einem Kopisten, der klatt für „kalt“ (20v, Z. 17) und Siczbe(n)czik für Septuaginta $\left(42^{v}, Z .16\right)$ setzt, deutsche Muttersprache zuzubilligen. Man könnte aber dagegensetzen, daß ein Tscheche nicht slawisch Sednu für lateinisch Semel gesetzt $\left(41^{\mathrm{v}}, \mathrm{Z}\right.$. 20) und kaum Had „Schlange“ mit Zad „Hinterteil“ verwechselt hätte $\left(50^{r}\right.$, Z. 2). Vielleicht war der Skribent Anfänger, die Arbeit eines Korrektors jedenfalls spricht nicht dagegen, und gelegentlich hat sogar der Rubrikator in den Kontext eingegriffen $\left(28^{\mathrm{v}}, \mathrm{Z}^{\mathrm{Z}}{ }^{17}, 31^{\mathrm{r}}, \mathrm{Z} .11,38^{\mathrm{v}}, \mathrm{Z} .4,39^{\mathrm{r}}, \mathrm{Z} .11\right.$ und 15, 43 v , Z. 11, 44 r, Z. 14, 45, Z. 4 und 5 u. a.). Zudem bestätigt ein Blick auf den vermutlich für Kaiser Maximilian geschriebenen Trialogus CVP 2868 - ihm ist der nächste Abschnitt unserer Arbeit gewidmet - mit gleichartig sinnstörenden Fehlern, daß der inhaltliche Standard der Glossare im 15. Jahrhundert sicher weniger geschätzt wurde als der formale. Vielleicht kam es u. a. deshalb zu solchen Mißlichkeiten, weil in den Kanzleien der Zeit buchstabengetreues Abschreiben nicht gefordert war ${ }^{136}$. Diese Freizügigkeit wird - zumindest in den deutschen Kolumnen unseres Textes - durch eine leichte Tendenz zur Buchstabenhäufung bestätigt, etwa B taylenn, Gotthaitt gegen A Teilen, Gotheit (14 $\left.{ }^{\mathrm{v}}, \mathrm{Z} .12 .17\right)$ usw.

In jedem Fall aber besteht eine gewisse Diskrepanz zwischen der doch routinierten Umsetzung der mitteldeutschen Vorlage ins Bairisch-Österreichische und den stümperhaften Fehlschreibungen des Kopisten. Leider läßt sich nicht absehen, ob und wie hier an eine sprachliche „Hintergrundarbeit“, etwa des Korrektors oder des Rubrikators, zu denken ist.

Der Trialogus wurde vorhin nicht zufällig angeführt, denn auch unser CVP 2945 dürfte für Maximilian angefertigt worden sein. Am 7. Februar 1507 läßt der Kaiser auf einem inventaryzedl eine Bestandsaufnahme von Urkunden, Büchern und gemalten Wappen machen, die in zwei Kisten der Burg von Wiener Neustadt lagern ${ }^{137}$. Darunter befindet sich Ain vocabulari, darinn Latein, Teutsch und Behemisch begriffen ist... Ernst Trenkler, der Bearbeiter der Zeit von 1368 bis 1519 in der monumentalen Hausgeschichte der Österreichischen Nationalbibliothek, hat - wie vor ihm Theodor Gottlieb - unsere Kopie des Ladislausvokabulars offensichtlich nicht gekannt und daher die Notiz im inventaryzedl auf den als Ambraser Handschrift geläufigen Trialogus von 1489 bezogen ${ }^{138}$. Dazu besteht allerdings kein zwingender Grund, denn es gibt, im Unterschied zu anderen Handschriften des Inventars, etwa Jacob Mennels Schachzabelbuch, keinen Hinweis, daß ein lateinisch-deutsch-tschechisches Glossar damals nach Innsbruck verbracht worden wäre ${ }^{139}$. Möglicherweise gilt eine Feststellung Al-

${ }^{136}$ L. E. Sснмгтт, Sprachgeschichte des Thüringisch-Obersächsischen im Spätmittelalter. Die Geschäftssprache von 1300 bis 1500 (Entstehung und Struktur der „,neuhochdeutschen Schriftsprache“ $1=$ Mitteldeutsche Forschungen 36/1). Köln-Graz 1966, 165.

137 Das Inventar ist zuletzt abgedruckt in Geschichte der Österreichischen Nationalbibliothek. Hrsg. von J. Stummoll 1 (Museion N. F. 2/3/1). Wien 1968, 29, Beil. IX.

138 Ebenda, 17, 23. Vgl. auch Th. GotTLieb, Büchersammlung Kaiser Maximilians I. Mit einer Einleitung über älteren Bücherbesitz im Hause Habsburg (Die Ambraser Handschriften. Beitrag zur Geschichte der Wiener Hofbibliothek 1). Leipzig 1900, 36.

139 Geschichte der Österreichischen Nationalbibliothek 1 (zit. A. 137) 23; GoTTLIEB, Büchersammlung (zit. A. 128) 41 . 
phons Lhotskys auch in unserem Zusammenhang: „Man hat sich in Wien ohnehin oft genug bei schwierigen Provenienzfragen die Sache bequem gemacht, indem man einfach ohne eigentliche Argumente vermutete, die entsprechende Handschrift sei eben aus Tirol gekommen" ${ }^{140}$.

Die erste Erwähnung unseres Vokabulars als Bestand der Hofbibliothek findet sich nicht im ältesten, 1576 angelegten Verzeichnis von Hugo Blotius, sondern erst im Katalog Sebastian Tengnagels unter Nr. 247 phil. als Vocabularium Lat. Germ. et Bohem. $4^{\circ}$ chart. ${ }^{141}$. Dies weist auf die Wende vom sechzehnten ins siebzehnte Jahrhundert. Dazu paßt genau ein Befehl Kaiser Rudolfs II., demzufolge zwischen 1577 und 1587 ein Teil der in Wiener Neustadt verbliebenen Bücher an die Wiener Hofbibliothek ging ${ }^{142}$.

Es bleibt die Frage, wann Maximilian oder seine Erzieher in den Besitz des kopierten Ladislausglossars gekommen sein könnten. Hiebei muß man sich vor Augen halten, wiesehr insgesamt die frühe Edukation des Kronprinzen auf Konzepten für Ladislaus Postumus aufbaute: Der sogenannte Ladislausdonat CVP 23* aus Melk, war - nach scholastischem Herkommen - überhaupt der erste Lehrbehelf des jüngeren Habsburgers ${ }^{143}$ und wir wissen nicht, welche Rolle das Melk-Tepler Ladislausdiurnale wirklich hatte ${ }^{144}$. Immerhin erlauben aber die Forschungen Alois Haidingers den Schluß, daß der zweite, in Böhmen verfertigte Teil dieses Kodex von derselben Hand stammt wie das Vokabular Holubařs. Es gibt also enge Verzahnungen. Sie rücken wieder das eingangs erwähnte Schreiben Hinderbachs von 1466 an Maximilians Mutter Leonore von Portugal in den Blickpunkt, dem eine Kopie des Piccolominitraktats für die Erziehung Ladislaus beigegeben war. Damals war Maximilian sieben Jahre alt. Sein vermutliches Studium der ars schlavonica wurde jedoch vorzeitig aufgegeben ${ }^{145}$. Dafür könnte auch sprechen, daß die restlichen 35 Blätter unserer Handschrift zwar rastriert aber nicht mehr ausgefüllt sind.

Diese unfreiwillige Unterbrechung könnte 1467 erfolgt sein, nachdem Eleonore gestorben war, Friedrich III. die Erziehung seines Sohnes selbst in die Hand nahm und bald dem konservativen informator latinitatis Peter Engelbrecht von Passail übertrug ${ }^{146}$. Vor diesem Jahr also dürfte für Zwecke der Prinzenerziehung die Kopie des Holubařvokabulars angefertigt worden sein, vielleicht in der kaiserlichen Residenz. Damals könnten auch Spezifika der böhmischen Illuminierungskunst die Kenntnis der böhmischen Fraktur und vielleicht auch die Idee, ein Tücheralphabet einzubauen, an den Hof von Wiener Neustadt gelangt sein. Am

140 A. Lhotsky, Die Bibliothek Kaiser Friedrichs III. MIÖG 58 (1950) 133.

141 CVP 9531, 157v.

142 Geschichte der Österreichischen Nationalbibliothek 1 (zit. A. 137) 123.

143 Fichtenau, Lehrbücher (zit. A.19) 9. Im Zusammenhang sei auch ein vor 1488 gedruckter Donat des Straßburger Druckers Johannes Weiß mit der Sign. 21.E.28 der Österreichischen Nationalbibliothek genannt, dessen erste Textseite illuminiert ist, und der mit der Devise AEIOU endet.

144 Vgl. den Katalog Die Kuenringer. Das Werden des Landes Niederösterreich. Zwettl 1981, Kat.Nr.203, beschrieben von Alois Haidinger. Noch nie genauer untersucht wurde die Bedeutung Melks im Zusammenhang mit Ladislaus und der Erziehung der Habsburger im fünfzehnten Jahrhundert, vgl. S. 71.

145 F. Scнмп, Eine neue Fassung der maximilianeischen Selbstbiographie. Diss Wien 1950, 6. Wie weit das semantische Feld Schlavonia etc. war, zeigt das Lexikon symphonon von Sigismund Gelenius, dessen vorwiegend tschechische Wortliste unter Sclavinica zusammengefaßt ist. Im Vorwort steht u. a.: Adhibitae sunt autem in hanc collationem quatuor linguae literatae duae, Graeca et Latina, duae barbarici soli, Germanica ac Sclauinica, nunc solae omnem Europam longe lateque occupantes. Dazu H. Brauner, Die tschechische Lexikographie des 16. Jahrhunderts. Breslau Diss. 1939, besonders 20, 25-27 und 31.

146 H. Fichtenau, Maximilian und die Sprache, in: Beiträge zur neueren Geschichte Österreichs (Veröffentlichungen des IfÖG 20 (1974) 33-34. 
Rande sei noch bemerkt, daß die Seiteneinteilung von A und B zu je zwanzig Zeilen bereits die Vocabula Francusiana für Wenzel IV. bestimmt hat, die auf S. 13 f. vorgestellt wurden.

Mit unserer Datierung stimmt überein, daß jene „Tafel“, CVP 2368, welche die Tücherbuchstaben enthält, noch zu Lebzeiten Eleonores angelegt worden ist ${ }^{147}$. Übrigens bleibt zu erwägen, ob das Fragmentarische und der etwas sorglose Kopialcharakter des CVP 2945 etwas mit der Umstellung des edukativen Programms und seinen Ursachen zu tun haben könnte. Ein bisher unbekanntes Zeugnis dieses Wechsels, auf das mich Frau Dr. Christine Glaßner hinwies, ist übrigens ein wortreicher Trialogus ad d(o)m(i)n(um) Maximilianu(m) adolescentem filiu(m) illustrissimi d(omi)ni ffriderici... aus dem Jahr 1470 im Melker Kodex 800. Er bezeichnet ein Streitgespräch zwischen sensualitas, voluntas und ratio und war nach Inhalt und Durchführung sicher nicht geeignet, auch nur das leiseste Interesse eines Elfjährigen zu erwecken.

Natürlich lassen sich die Angaben über herrscherliche Sprachkompetenzen umso weniger überprüfen, je weiter man in die Geschichte zurückgeht ${ }^{148}$, und gerade Kaiser Maximilians Selbststilisierung zum polyglotten Fürstenideal macht mißtrauisch. Trotzdem muß im Zusammenhang v. a. das Kapitel Wie der jung weiß kunig von einem pauren windisch und behamisch lernte ${ }^{149}$ aus dem Weißkunig erwähnt werden, weil es sogar von heimlichen Sprachübungen berichtet: Dieweil der jung weiß kunig bey seinem vater, dem alten weißn kunig was, da kam gar oft ain pawr zu ime, der ime albegen seltzame frucht pracht. Nun kunt derselb pauer windisch und behamisch; da understund sich der jung weiß kunig, von dem pawrn in gehaim dieselben zwo sprachen zu lernen. Derselb pawr kunt sich gegen dem jungen weisen kunig mit reden in sonderhait wol schicken, dardurch der jung kunig die zwo sprach dermassn begriff, das er die verstund und, wiewol er dieselb sprach gar wenig prauchet, so vergaß er doch der nit, sonder wann man dieselbn sprach redet, so verstund er die nach seiner notturft. Für Maximilian war dieses Detail immerhin wichtig genug, es mit einem zugehörigen Holzschnitt - möglicherweise von Hans Burgkmayer und ohne neuerliche Nennung von behamisch - zu verdeutlichen (Abb. 41).

Für die hermetische Verwendung ad usum delphini spricht noch, daß die beiden Handschriften mit dem von Johann Holubař kompilierten Text keinerlei Nachfolge fanden. Dazu gibt es eine bezeichnende Parallele im Wirken des tschechischen Erziehers Rafael Mnišofskys für Ferdinand III. im Jahr $1628^{150}$.

147 Fichtenau, Lehrbücher (zit. A. 19) 17.

148 Dazu T. Berger, Tschechischunterricht in der Habsburgerfamilie ab 1526. Wiener Slavistisches Jahrbuch $46(2000) 62$.

149 Weißkunig. Nach den Dictaten und eigenhändigen Aufzeichnungen Kaiser Maximilians I. zusammengestellt von Marx Treitzsauerwein von Ehrentreitz, hrsg. von A. Schultz (JKhSW 6). Wien 1888, 74.

150 Vgl. FlajšHans, Klaret 1 (zit. A. 75) XXXI. 